\title{
Two New Considerations for Improving the Diagnosis of a1-Antitrypsin Deficiency-Associated Liver Disease
}

\author{
David H. Perlmutter $\cdot$ Kapil Chopra • \\ Andrew Chu
}

Published online: 30 January 2015

(c) Springer Science+Business Media New York 2015

The study by Tapper et al. [1], published in this issue of Digestive Diseases and Sciences, rather starkly emphasizes the need for improvements in clinical practice used in diagnosing the liver disease caused by $\alpha 1$-antitrypsin deficiency (ATD). Due to its rarity and because it is more frequently encountered as a cause of chronic obstructive pulmonary disease (COPD), ATD is not common for hepatologists to encounter. Discovered in three patients with COPD in 1963 [2], ATD was diagnosed some years later in an infant with liver disease [3]. In 1986, Eriksson et al. [4] described ATDrelated adult-onset liver cirrhosis and hepatocellular carcinoma. We now know that the "classical" form of ATD, homozygosity for the $\alpha 1$-antitrypsin Z (ATZ) allele, affects $\sim 1$ in 3,000 live births and distinct subgroups of this population are affected by COPD and/or liver disease (reviewed in 5). The underlying genetic defect is a point mutation that leads to protein misfolding with resultant intracellular accumulation of ATZ monomers and polymers accompanied by a marked reduction in ATZ secretion, such that circulating levels are $85-90 \%$ lower than normal. Since $\alpha 1$-antitrypsin (AT) is a serine protease inhibitor, particularly a neutrophil elastase inhibitor, the reduced circulating levels of AT facilitate

D. H. Perlmutter $(\bowtie) \cdot$ A. Chu

Department of Pediatrics, University of Pittsburgh School of Medicine, Children's Hospital of Pittsburgh and University of Pittsburgh Medical Center, Pittsburgh, PA, USA

e-mail: David.Perlmutter@chp.edu

A. Chu

e-mail: andrew.chu@chp.edu

\section{K. Chopra}

Department of Medicine, University of Pittsburgh School of Medicine, University of Pittsburgh Medical Center, Pittsburgh, PA, USA

e-mail: choprakb3@upmc.edu proteolytic damage of the pulmonary connective tissue matrix by a loss-of-function mechanism. In contrast, liver disease is caused by the proteotoxic effects of misfolded ATZ that accumulates in hepatocytes, a gain-of-toxic function mechanism [5]. Several drugs, some of which are currently approved for other indications, such as carbamazepine, by enhancing the intracellular degradation of misfolded ATZ can reverse liver damage in a mouse model of ATD [6,7] and thus are being tested clinically. Gene silencing techniques can reverse the liver damage in the ATD mouse model [8]. Furthermore, genomic editing techniques that would potentially correct the underlying genetic defect and therein prevent both liver and lung disease appear to be possible in the near future $[9,10]$.

The liver disease caused by ATD is characterized by predominant fibrosis and cirrhosis with relatively little inflammation. Although ATD is best known for liver disease in infants, children, and adolescents, ATD also causes cirrhosis and hepatocellular carcinoma in adults with a peak age of 50-65 years. There are still many mysteries about the epidemiology of liver disease in people affected by this genetic deficiency. For one, a landmark study that tracked a cohort of homozygotes from an unbiased newborn screening initiative in Sweden has reported that only a subgroup developed clinically significant liver disease [11], stressing the importance of genetic and environmental modifiers in determining disease phenotype [5]. Since the cohort has yet to reach the peak age range of 50-65 years, the exact proportion of the population affected with liver disease may be larger than what is currently described. It is also still not clear whether heterozygotes for the ATZ allele are predisposed to fibrotic liver disease or to hepatic cancer. A number of studies and clinical experiences have suggested that ATZ heterozygotes, perhaps even homozygotes, may be more likely to have liver disease if they also have other causes of liver disease, including cystic fibrosis, nonalcoholic 
steatohepatitis, and hemochromatosis, among others [12]. We recently noticed that more than $85 \%$ of liver transplants performed in the USA for a diagnosis of ATD were in adults with peak age 50-65 years, but an unpublished analysis of the liver transplantations performed for this disease at the University of Pittsburgh Medical Center indicates that only a proportion of these were ATZ homozygotes, and many carried a diagnosis of other diseases that could have caused or contributed to the progression of liver disease.

In their study entitled "Low yield and utilization of confirmatory testing in a cohort of patients with liver disease associated with alpha-1-antitrypsin deficiency," Tapper et al. [1] retrospectively investigated the results of serum AT measurements in 4,985 patients performed at a large urban clinical laboratory. Low levels of AT were present in 73 patients $(1.4 \%)$ with a definitive ATD diagnosis made in eight patients $(0.16 \%)$. Nonetheless, only 23 of the 73 (31.5\%) patients with low AT levels had the appropriate testing for AT PI phenotype (determined by isoelectric focusing of the AT protein in serum). Utilization of the PI phenotype test was inversely correlated with testing for autoimmune hepatitis, hepatitis B (HBV), hepatitis C (HCV), and Wilson's disease. Patients with low AT levels had a significantly more frequent history of comorbid pulmonary disease and a higher incidence of ALT levels $<40$. The authors concluded that using serum AT levels to evaluate elevated ALT levels is low-yield, that ATD is frequently evaluated simultaneously with evaluations for autoimmune hepatitis, HBV, and HCV, and that PI phenotypic confirmation of ATD in patients with low AT levels was underutilized. According to their analysis in which eight ATD patients are identified from 4,985 serum AT determinations (or from 4,030 if hepatitis cases are excluded), the cost of this test to diagnose a single case of ATD is $\$ 97,811(\$ 79,128)$. Due to its retrospective design, there are some missing data concerning the patient population and clinical decision making, in particular the motivation for selecting diagnostic tests such as serum AT levels, the sequence used for ordering tests for other causes of liver disease, and the rate of liver biopsy. Furthermore, the cost of diagnosing ATD is based on the eight patients identified, which may be an underestimate since only 23 of the 73 patients with low AT levels had PI phenotype testing, perhaps due to other diagnoses being made such as nonalcoholic fatty liver disease (NAFLD), alcohol, hemochromatosis, HCV, Gilbert's, or primary biliary cholangitis, or the assumption that a low AT level was diagnostic of ATD. It is possible that some of the 50 patients who did not have the appropriate confirmatory testing actually had the "classical" form of ATD and some could have had both ATD and one of the other causes of liver disease. This is no longer an academic consideration because these patients may become candidates for the new therapies currently in clinical trials. In particular, 25 patients with low AT levels were diagnosed with NAFLD, which can coexist with the "classical" form of ATD in patients with severe liver disease. One of the interventions being considered for ATDrelated liver disease, namely autophagy enhancer drugs, may also be effective in NAFLD because autophagy has recently been reported to mediate intracellular fat clearance, a process now known as lipophagy [13]. It is also possible that some of the 50 patients who did not have confirmatory testing were heterozygous for the ATZ allele and we would argue that the definitive diagnosis of heterozygosity for the ATZ allele will become more important in the future. Although at this time there is no clear evidence that ATZ heterozygosity causes liver disease by itself, we believe that this is most likely due to limitations in the way this causality issue can be investigated in a human population and not due to any data that clearly refute the causal association. Numerous reports exist of patients with severe liver disease and ATZ heterozygosity with no other explanation for their liver disease, and many of these undergo liver transplantation in the USA each year. It is our hope that this issue will be addressed in the near future by new drugs and therapeutic strategies currently under investigation that are safe enough to test in this ATZ heterozygote population.

Tapper et al. report that four patients were diagnosed with ATD on the basis of liver histology. Presumably, this means that these patients had the intrahepatocytic inclusions that are characteristic of ATD. However, intrahepatocytic inclusions positive for PAS/diastase and AT antibody staining have been described in patients who do not have ATD [5]. Moreover, intracellular inclusions may be scant and underappreciated in occasional patients with the $\mathrm{ZZ}$ phenotype and all of the other clinicopathological characteristics of the classical form of ATD [5]. For this reason, we believe that liver biopsy is not sufficient in and of itself to make the diagnosis of ATD liver disease and should always be combined with a PI phenotype determination.

Lastly, the authors wisely point out that their study cannot address the generalizability of the population and testing patterns used by the study clinicians. Nevertheless, the main conclusion from this important report, that clinical practice guidelines for the diagnosis of ATD-related liver disease are needed immediately, is inarguable. Not only will this improve the quality and the costs of care but it will be essential for identifying the appropriate patients for new therapies as they become available and for carrying out clinical trials to determine the efficacy of these new therapies.

\section{References}

1. Tapper EB, Patwardhan VR, Curry MP. Low yield and utilization of confirmatory testing in a cohort of patients with liver disease 
assessed for alpha-1-antitrypsin deficiency. Dig Dis Sci. (Epub ahead of print). doi:10.1007/s10620-014-3490-y.

2. Laurell C-B, Eriksson S. The electrophoretic $\alpha 1$-globulin pattern of serum in $\alpha 1$-antitrypsin deficiency. Scand J Clin Lab Invest. 1963;15:132-140.

3. Sharp HL, Bridges RA, Krivit W, Freier EF. Cirrhosis associated with alpha-1-antitrypsin deficiency: a previously unrecognized inherited disorder. J Lab Clin Med. 1969;73:934-939.

4. Eriksson S, Carlson J, Velez R. Risk of cirrhosis and primary liver cancer in alphal-antitrypsin deficiency. $N$ Engl $J$ Med. 1986;314:736-739.

5. Ghouse R, Chu A, Wang Y, Perlmutter DH. Mysteries of a1antitrypsin deficiency: emerging therapeutic strategies for a challenging disease. Dis Model Mech. 2014;7:411-419.

6. Hidvegi T, Ewing M, Hale P, et al. An autophagy-enhancing drug promotes degradation of mutant alpha1-antitrypsin $\mathrm{Z}$ and reduces hepatic fibrosis. Science. 2010;329:229-232.

7. Li J, Pak SC, O'Reilly LP, et al. Fluphenazine reduces proteotoxicity in C. elegans and mammalian models of alpha-1-antitrypsin deficiency. PLoS One. 2014;9:e87260.
8. Guo S, Booten SL, Aghajan M, et al. Antisense oligonucleotide treatment ameliorates alpha-1 antitrypsin-related liver disease in mice. J Clin Invest. 2014;124:251-261.

9. Yusa K, Rashid ST, Strick-Marchand H, et al. Targeted gene correction of $\alpha 1$-antitrypsin deficiency in induced pluripotent stem cells. Nature. 2011;478:391-394.

10. Long C, McAnally JR, Shelton JM, Mireault AA, Bassel-Duby R, Olson EN. Prevention of muscular dystrophy in mice by CRISPR/Cas9mediated editing of germline DNA. Science. 2014;345:1184-1188.

11. Bernspång E, Carlson J, Piitulainen E. The liver in 30-year-old individuals with alpha1-antitrypsin deficiency. Scand J Gastroenterol. 2009;44:1349-1355.

12. Regev A, Guaqueta C, Molina EG, et al. Does the heterozygous state of alpha-1 antitrypsin deficiency have a role in chronic liver diseases? Interim results of a large case-control study. J Pediatr Gastroenterol Nutr. 2006;43:S30-S35.

13. Liu K, Czaja MJ. Regulation of lipid stores and metabolism by lipophagy. Cell Death Diff. 2013;20:3-11. 